\title{
Compact 2-D FDTD Method Combined With Padé Approximation Transform for Leaky Mode Analysis
}

\author{
Qiaoyin Lu, Weihua Guo, Diarmuid C. Byrne, and John F. Donegan, Senior Member, IEEE
}

\begin{abstract}
Leaky mode analysis has been carried out based on the compact 2-D finite-difference time-domain (FDTD) method combined with the uniaxial anisotropic perfectly matched-layer (UPML) absorption boundary condition and the Padé approximation transform technique. The imaginary part of the effective index of these leaky modes can be calculated independent of the real part, so very small leaky loss can be calculated reliably based on the proposed scheme. Mode coupling effects have also been accounted for naturally within the scheme because the simulation is carried out in the time domain and is full-vectorial. Leaky modes in the ARROW waveguide and the deep ridge waveguide have been analyzed by the proposed scheme. Leakage cancellation behavior for high-order leaky modes in very deeply etched ridge waveguides at specific ridge widths has been observed.
\end{abstract}

Index Terms-Compact 2-D finite-difference time-domain (FDTD) method, leaky mode analysis, Padé approximation transform (PAT), uniaxial perfectly matched layer (UPML).

\section{INTRODUCTION}

A NALYSIS of leaky modes in optical waveguides is indispensable for designing optical waveguide-based devices. A group of numerical methods have been proposed to solve leaky modes, such as the spectral index scheme [1], the eigenequation solving method based on the finite-element scheme [2], [3], the finite-difference method [4], and the imaginary beam propagation method based on the finite-element scheme [5], [6]. A method based on real propagation has also been used to estimate the leaky loss [7]. A common feature of these methods is that they search the complex effective indexes of the leaky modes in the complex plane through some iteration processes and are generally initial-guess-sensitive. Generally, the leaky loss is calculated from the imaginary part of the leaky mode effective index, which is rather challenging, especially for leaky modes with very low leakage loss. This is because the imaginary part of the effective index is generally several orders of magnitude smaller than the real part. For example, even when the leaky loss reaches a level of $100 \mathrm{~dB} / \mathrm{cm}$, the imaginary part is still just $\sim 3.0 \times 10^{-4}$ at $1550 \mathrm{~nm}$, which is four orders of magnitude smaller than the real part, which is generally around 3.2 .

The finite-difference time-domain (FDTD) technique is a powerful tool for modal analysis. Unlike the frequency-domain

Manuscript received January 26, 2010; accepted March 29, 2010. Date of publication April 12, 2010; date of current version May 26, 2010. This work was supported by the Science Foundation Ireland PIFAS under Grant 07/SRC/I1173.

The authors are with the Semiconductor Photonics Group, School of Physics, Trinity College, Dublin 2, Ireland (e-mail: luqi@tcd.ie).

Color versions of one or more of the figures in this paper are available online at http://ieeexplore.ieee.org.

Digital Object Identifier 10.1109/JLT.2010.2048011 modal solvers mentioned above, it solves Maxwell's equations in the time domain through explicit iterations, and the broadband frequency domain information is naturally involved. However, it requires large memory and computation time for 3-D simulations. In [8], the compact 2-D FDTD was first proposed and has been widely employed to analyze uniform waveguide structures in the microwave regime. By introducing a variable transform, the 2-D FDTD simulation can run with real variables and thus save the computation effort further [9]. Recently, we employed a model which combined the compact 2-D FDTD technique with the Padé approximation transform to analyze high-order leaky modes in deep ridge semiconductor optical waveguides [10]. This model is simple to implement, and it can provide reliable analysis because it calculates the real part and imaginary part of the mode effective index separately, which is a distinct advantage, especially when analyzing leaky modes with very low leaky loss.

To employ the compact FDTD method to analyze leaky modes in optical waveguides, two points are crucial: one is the absorption boundary condition ( $\mathrm{ABC})$; the other is the transforming tool which changes the FDTD data from the time domain to the frequency domain. For the $\mathrm{ABC}$, we use the perfectly matched-layer (PML) [11] absorption condition which has been shown to have superior performance over other kinds of ABCs. To facilitate the extension of the PML from 3-D into 2-D, the so-called uniaxial anisotropic perfect matched layer (UPML) [12] boundary conditions were adopted to efficiently terminate the FDTD computation window. In the compact 2-D FDTD method, the waveguide is treated as a 2-D cavity of which the resonant modes represent the 3-D propagation modes of the waveguide. By transforming the FDTD data from the time domain to the frequency domain, the corresponding mode spectra can be calculated, from which the mode information can be extracted. The fast Fourier transform (FFT) is the generally used tool for this transformation, however, it is inefficient to obtain high-frequency resolution. To reduce the FDTD simulation time, the efficient Padé approximation transform (PAT) [13] is used to produce high spectral resolution through short FDTD data sequences. Through the PAT technique, the mode spectra can be calculated, and then a Lorentzian fitting can be used to extract the mode information. Because this approach is based on the time-domain simulation, it takes into account all mode coupling effects naturally. For frequency-domain methods, it could be more difficult to account for the mode-coupling effect, although they could be more efficient because they analyze a specific mode for a single run.

In this paper, we will introduce this proposed method in detail and employ it to analyze leaky modes in ARROW waveguides and in deep ridge waveguides. The paper is organized as follows. In Section II, the 2-D FDTD method with the UPML boundary 
condition and the PAT technique are introduced. In Section III, a metal waveguide which has analytical solutions to its mode has been simulated by the proposed method with results compared with analytical results. In Section IV, leaky modes in ARROW waveguides and in deep ridge waveguides are analyzed by this method. In Section V, conclusions are given.

\section{THEORY}

Using the compact 2-D FDTD for mode analysis, we first choose a propagation constant $\beta$ along with a Gaussian excitation pulse for the field within the waveguide core. Then, we run the FDTD algorithm to update the field both in space and in time, and, subsequently, the field at some points in the waveguide is recorded during the iteration. Finally, the recorded field time data are converted into the frequency domain by the PAT technique and the frequency spectrum is obtained at which the propagation modes in the waveguide exhibit the selected $\beta$.

\section{A. Compact 2-D FDTD With UPML}

The compact 2-D FDTD is a simplification of 3-D FDTD under the assumption that all of the electromagnetic fields depend on the propagation direction $z$ simply as $\exp (-j \beta z)$, where $\beta$ is the propagation constant. This is justified for waveguide structures that are uniform in the propagation direction. In compact 2-D FDTD, all electromagnetic fields are represented by a 2-D mesh, where $H_{x}\left(E_{y}\right)$ and $H_{y}\left(E_{x}\right)$ occupy the same mesh points [8].

The PML ABC has been demonstrated as the most effective boundary condition to truncate FDTD lattices. Among the various modifications and extensions of the PML, the so-called UPML [12], usually denoted as unsplit PML, has the advantage of performing as well as split PML while maintaining Maxwell's equations in the physical form by the use of uniaxial anisotropic lossy material. In the UPML regions, there are 12 equations of which six are related to electric fields $\vec{E}$ and magnetic fields $\vec{H}$; six are related to electric flux densities $\vec{D}$ and magnetic flux densities $\vec{B}$. In the compact 2-D FDTD, the PML conductivity along the propagation direction $\sigma_{z}$ equals zero, hence the time-domain differential equations can be written as

$$
\begin{aligned}
& \left\{\begin{array}{l}
\varepsilon \frac{\partial E_{x}}{\partial t}=\frac{\partial D_{x}}{\partial t}+\frac{\sigma_{x}}{\varepsilon} D_{x} \\
\varepsilon \frac{\partial E_{y}}{\partial t}+\sigma_{x} E_{y}=\frac{\partial D_{y}}{\partial t}+\frac{\sigma_{y}}{\varepsilon} D_{y} \\
\varepsilon \frac{\partial E_{z}}{\partial t}+\sigma_{y} E_{z}=\frac{\partial D_{z}}{\partial t} \\
\mu_{0} \frac{\partial H_{x}}{\partial t}=\frac{\partial B_{x}}{\partial t}+\frac{\sigma_{x}}{\varepsilon} B_{x} \\
\mu_{0} \frac{\partial H_{y}}{\partial t}+\frac{\mu}{\varepsilon} \sigma_{x} H_{y}=\frac{\partial B_{y}}{\partial t}+\frac{\sigma_{y}}{\varepsilon} B_{y} \\
\mu_{0} \frac{\partial H_{z}}{\partial t}+\frac{\mu}{\varepsilon} \sigma_{y} H_{z}=\frac{\partial B_{z}}{\partial t}
\end{array}\right. \\
& \left\{\begin{array}{l}
\frac{\partial D_{x}}{\partial t}=\beta H_{y}+\frac{\partial H_{z}}{\partial y}-\frac{\sigma_{y}}{\varepsilon} D_{x} \\
\frac{\partial D_{y}}{\partial t}=-\beta H_{x}-\frac{\partial H_{z}}{\partial x} \\
\frac{\partial D_{z}}{\partial t}=\frac{\partial H_{y}}{\partial x}+\frac{\partial H_{x}}{\partial y}-\frac{\sigma_{x}}{\varepsilon} D_{z} \\
\frac{\partial B_{x}}{\partial t}=\beta E_{y}-\frac{\partial E_{z}}{\partial y}-\frac{\sigma_{y}}{\varepsilon} B_{x} \\
\frac{\partial B_{y}}{\partial t}=-\beta E_{x}+\frac{\partial E_{z}}{\partial x} \\
\frac{\partial B_{z}}{\partial t}=\frac{\partial E_{x}}{\partial y}-\frac{\partial E_{y}}{\partial x}-\frac{\sigma_{x}}{\varepsilon} B_{z}
\end{array}\right.
\end{aligned}
$$

where $\varepsilon=\varepsilon_{0} \varepsilon_{r}$ is the permittivity, $\mu_{0}$ is the permeability, and $\sigma_{x}\left(\sigma_{y}\right)$ is the conductivity in the upper and lower (right) PML (as shown in Fig. 2). Subsequently, the time-dependent field components can be computed via the standard explicit compact 2-D FDTD update expressions

$$
\begin{aligned}
D_{x}^{n+1}= & \frac{1-\sigma_{y} \Delta t / 2 \varepsilon}{1+\sigma_{y} \Delta t / 2 \varepsilon} D_{x}^{n}+\frac{\Delta t}{1+\sigma_{y} \Delta t / 2 \varepsilon} \frac{\partial H_{z}^{n+0.5}}{\partial y} \\
& +\frac{\beta \Delta t}{1+\sigma_{y} \Delta t / 2 \varepsilon} H_{y}^{n+0.5} \\
D_{y}^{n+1}= & D_{y}^{n}-\Delta t \frac{\partial H_{z}^{n+0.5}}{\partial x}-\beta \Delta t H_{x}^{n+0.5} \\
D_{z}^{n+1}= & \frac{1-\sigma_{x} \Delta t / 2 \varepsilon}{1+\sigma_{x} \Delta t / 2 \varepsilon} D_{z}^{n}+\frac{\Delta t}{1+\sigma_{x} \Delta t / 2 \varepsilon} \frac{\partial H_{y}^{n+0.5}}{\partial x} \\
& -\frac{\Delta t}{1+\sigma_{x} \Delta t / 2 \varepsilon} \frac{\partial H_{x}^{n+0.5}}{\partial y} \\
E_{x}^{n+1}= & E_{x}^{n}+\frac{1+\sigma_{x} \Delta t / 2 \varepsilon}{\varepsilon} D_{x}^{n+1}-\frac{1-\sigma_{x} \Delta t / 2 \varepsilon}{\varepsilon} D_{x}^{n} \\
E_{y}^{n+1}= & \frac{1-\sigma_{x} \Delta t / 2 \varepsilon}{1+\sigma_{x} \Delta t / 2 \varepsilon} E_{y}^{n}+\frac{1+\sigma_{y} \Delta t / 2 \varepsilon}{\varepsilon\left(1+\sigma_{x} \Delta t / 2 \varepsilon\right)} D_{y}^{n+1} \\
& -\frac{1-\sigma_{y} \Delta t / 2 \varepsilon}{\varepsilon\left(1+\sigma_{x} \Delta t / 2 \varepsilon\right)} D_{y}^{n} \\
E_{z}^{n+1}= & E_{z}^{n}+\frac{1}{\varepsilon\left(1+\sigma_{y} \Delta t / 2 \varepsilon\right)} D_{z}^{n+1}-\frac{1}{\varepsilon\left(1+\sigma_{y} \Delta t / 2 \varepsilon\right)} D_{z}^{n} \\
B_{x}^{n+1.5}= & \frac{1-\sigma_{y} \Delta t / 2 \varepsilon}{1+\sigma_{y} \Delta t / 2 \varepsilon} B_{x}^{n+0.5}-\frac{\Delta t}{1+\sigma_{y} \Delta t / 2 \varepsilon} \frac{\partial E_{z}^{n+1}}{\partial y} \\
& +\frac{\beta \Delta t}{1+\sigma_{y} \Delta t / 2 \varepsilon} E_{y}^{n+1} \\
& -\frac{1}{\mu \varepsilon\left(1+\sigma_{y} \Delta t / 2 \varepsilon\right)} B_{z}^{n+0.5} \\
B_{y}^{n+1.5}= & B_{y}^{n+0.5}+\Delta t \frac{\partial E_{z}^{n+1}}{\partial x}+\beta \Delta t E_{x}^{n+1} \\
B_{z}^{n+1.5}= & \frac{1-\sigma_{x} \Delta t / 2 \varepsilon}{1+\sigma_{x} \Delta t / 2 \varepsilon} B_{z}^{n+0.5}-\frac{\Delta t}{1+\sigma_{x} \Delta t / 2 \varepsilon} \frac{\partial E_{y}^{n+1}}{\partial x} \\
& +\frac{\Delta t}{1+\sigma_{x} \Delta t / 2 \varepsilon} \frac{\partial E_{x}^{n+1}}{\partial y} \\
H_{z}^{n+1.5}= & \frac{1-\sigma_{y} \Delta t / 2 \varepsilon}{1+\sigma_{y} \Delta t / 2 \varepsilon} H_{z}^{n+0.5}+\frac{1-\sigma_{y} \Delta t / 2 \varepsilon}{\mu} B_{y}^{n+0.5} B_{z}^{n+1.5} \\
H_{x}^{n+1.5}= & H_{x}^{n+0.5}+\frac{1+\sigma_{x} \Delta t / 2 \varepsilon}{\mu} B_{x}^{n+1.5}-\frac{1-\sigma_{x} \Delta t / 2 \varepsilon}{\mu} B_{x}^{n+0.5} \\
H_{y}^{n+1.5}= & \frac{1-\sigma_{x} \Delta t / 2 \varepsilon}{1+\sigma_{x} \Delta t / 2 \varepsilon} H_{y}^{n+0.5}+\frac{1+\sigma_{y} \Delta t / 2 \varepsilon}{\mu \varepsilon\left(1+\sigma_{x} \Delta t / 2 \varepsilon\right)} B_{y}^{n+1.5}
\end{aligned}
$$

where the spatial differentiation is not expanded for compactness. The stability of the compact 2-D FDTD algorithm is ensured by choosing the time step $\Delta t$ to satisfy the stability criterion [14]

$$
\Delta t \leq \frac{1}{\sqrt{2 / \Delta s^{2}+\beta^{2} / 4}}
$$

where $\Delta s$ is the spatial step used for the spatial discretization which however has not been shown in (2). A uniform spatial mesh has been assumed. To reduce the pseudo-reflection caused by discretization, the PML conductivity $\sigma_{x}\left(\sigma_{y}\right)$ is defined to have a polynomial graded profile, which does not vary with $y(x)$ but does vary with $x(y)$ as $\sigma_{x}\left(\sigma_{y}\right)=(\delta / d)^{m} \sigma_{\max }$, where $\delta$ represents the distance between a point inside the PML and the inner interface of the PML, $d$ is the thickness of the PML, $m=2$ is the order of the polynomial variation, and $\sigma_{\max }=$ $(m+1) \varepsilon_{r}^{1 / 2} /(150 \pi \Delta s)$ is the optimized maximum conductivity [12]. In the simulation, the PMLs should be placed far away from the waveguide core region to ensure that their effects on the mode properties can be negligible.

\section{B. Padé Approximation Transform}

In order to obtain mode frequencies and quality factors ( $Q$-factors), the FDTD output in the time-domain must be transformed into the frequency domain. The generally used tool is the fast Fourier transform (FFT), however, the resolution of the FFT scheme is inversely proportional to the total persistence time of the FDTD iteration, i.e., the product of the 
iteration number and the time step. The time step of the FDTD technique is limited by the Courant limit as demonstrated in (5), so the FDTD iteration number is required to be very large. If the propagation loss is very small (i.e., high $Q$-factor for the leaky modes), a very long time FDTD simulation is needed for FFT to obtain accurate mode $Q$-factors and frequencies.

The Padé approximation technique which uses diagonal Padé approximants to approximate the discrete Fourier transform (DFT) of infinite FDTD data sequences, can obtain high frequency resolution based on relatively short FDTD data sequences [13], which can greatly save on the FDTD running time.

For the infinite time response of an electromagnetic field component $u(n \Delta t)(n=0 \sim \infty)$, we can introduce a function of variable $z$ as

$$
\begin{aligned}
F(z, f) & =\sum_{n=0}^{\infty} C_{n} z^{n} \\
C_{n} & =u(n \Delta t) \exp (-j 2 \pi f n \Delta t) .
\end{aligned}
$$

It is obvious that $F(1, f)$ is the discrete Fourier transform of $u(n \Delta t)$

$$
U(f)=\sum_{n=0}^{\infty} u(n \Delta t) \exp (-j 2 \pi f n \Delta t)
$$

Practically through FDTD simulations, we can just get a finite time response $u(n \Delta t)(n=0 \sim N, N$ is the number of time steps.). The function $F(z, f)$ at a certain frequency can be approximated by its diagonal Padé approximant $[N / 2, N / 2](z)$, which can be efficiently calculated using Baker's algorithm. Applying this Padé approximation to all the frequencies in the interested frequency range we can obtain the frequency spectrum. To save the computation time of the Padé approximant, generally the original FDTD output needs to be filtered and decimated to reduce $N$ [13].

Through the PAT technique the mode spectra can be calculated. A Lorentzian fitting can be used to find the mode frequency $f_{0}\left(\lambda_{0}\right)$ and the $3-\mathrm{dB}$ bandwidth $\Delta f(\Delta \lambda)$. The mode quality factor can be calculated as $Q=f_{0} / \Delta f$. Then the propagation loss (imaginary part of the mode effective index) of the waveguide mode at $f_{0}$ is given by [15]

$$
\alpha=\frac{k_{0} n_{g}}{Q}, n_{i}=\frac{n_{g}}{2 Q} .
$$

The real part of the mode effective index can be calculated from $n_{\mathrm{eff}}=\beta / k_{0}$, where $k_{0}$ is the free-space wave number, and $n_{g}$ is the group index of the waveguide mode, which is calculated from the $\mathrm{n}-\lambda$ dispersion curve

$$
n_{g}=n_{\mathrm{eff}}-\lambda \frac{d n_{\mathrm{eff}}}{d \lambda} .
$$

\section{Mode Field Distribution}

After the mode frequency being extracted from the mode spectrum by PAT for a given $\beta$, the mode field distribution can be calculated through DFT by running the FDTD simulation again.

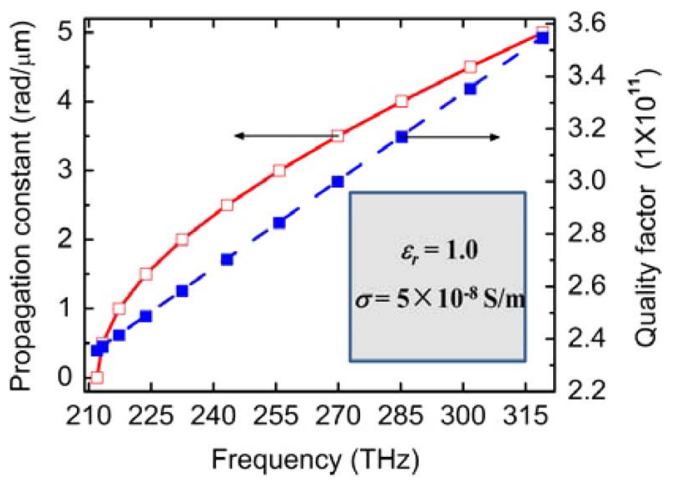

Fig. 1. Propagation constant and quality factor of the fundamental mode versus mode frequency in a metallic waveguide as shown in the inset, calculated by the compact 2-D FDTD method and the PAT technique (dots) and by the analytical formula (line).

We can build the DFT into the FDTD solver, so that we can perform the DFT while updating the FDTD iterations. For the mode with the frequency $f_{0}$, the DFT is defined as [16]

$$
\Phi\left(p, q, f_{0}\right)=\sum_{n=0}^{N-1} \varphi(p, q, n) \exp \left(-j 2 \pi f_{0} n \Delta t\right)
$$

where $n$ is the number of time steps, $(p, q)$ is the mesh point in the computational window, $\Delta t$ is the time step, and $\varphi$ represents an electromagnetic field component.

\section{MODEL VALIDATION}

Before using the approach to analyze leaky modes in real semiconductor waveguides (as in such cases the leaky loss could be quite low), first we demonstrate that the PAT technique can efficiently analyze very low level of modal losses. For this validation a perfect metal waveguide with the mode quality factor as high as $1 \times 10^{11}$ is considered. The structure we simulated is a square waveguide with the side length $a$ of $1.0 \mu \mathrm{m}$ formed by perfect metals and filled with a slightly lossy medium $\left(\varepsilon_{r}=\right.$ $1.0, \sigma=5 \times 10^{-8} \mathrm{~S} / \mathrm{m}$ ), as shown in the inset of Fig. 1. The mode frequency and quality factor of the fundamental mode of this metal waveguide can be calculated analytically using the following formula:

$$
\left\{\begin{array}{l}
f_{0,0}=\sqrt{\frac{c^{2}}{\varepsilon_{r}}\left(\frac{1}{2 a^{2}}+\frac{\beta^{2}}{4 \pi^{2}}\right)-\frac{\sigma^{2}}{16 \pi^{2} \varepsilon_{0}^{2} \varepsilon_{r}^{2}}} \\
Q=\frac{2 \pi f_{0,0} \varepsilon_{0} \varepsilon_{r}}{\sigma}
\end{array}\right.
$$

where $c$ is the light velocity in free space. The compact 2-D FDTD method is used to simulate the field evolution in the 2-D cavity formed by the waveguide, and the PAT technique is used to calculate the mode spectrum from which the mode frequencies and quality factors are extracted. The discrete space cell used in the simulation has a dimension of $0.01 \mu \mathrm{m}$ and the FDTD program is generally iterated in the time domain by 10 000 steps after the pulse finished. The obtained mode frequencies and quality factors of the fundamental mode are plotted in Fig. 1, which agrees well with the analytical results. A typical example is that, when $\beta=0$, the mode frequency and quality factor of the fundamental mode calculated by this model are $211.982 \mathrm{THz}$ and $2.355 \times 10^{11}$, respectively, which are in 
good agreement with the theoretical values: $211.985 \mathrm{THz}$ and $2.359 \times 10^{11}$.

\section{NUMERICAL ANALYSIS}

The waveguides we analyze in the following have mirror symmetry with respect to the waveguide axis, so the symmetry or anti-symmetry conditions can be added to the electric field components at the symmetry plane to excite the even and odd modes, respectively, which will save the computation domain by half and thus save the computation time greatly. The Gaussian function modulated cosine impulse is added to the field at several arbitrary points inside the waveguide core as the exciting source along with the proper propagation constant $\beta_{0}$. The excitation is added to the $E_{x}$ component which is the major electric field. We avoid those high symmetry points in our selection because at these positions the specific mode field could be zero. Several modes can be excited simultaneously. The time variations of the field component at several selected points different from those excitation points inside the waveguide core are recorded as the FDTD output. Then we calculate the field spectrum from the FDTD output by the Padé approximation transform. We can use any field component and get same results for the calculation.

Using the compact 2-D FDTD we can calculate the mode information at a given propagation constant $\beta$, which means the analyzed mode is operated at the wavelength $\lambda$. To analyze the mode at a specific wavelength $\lambda_{0}$, the proper propagation constant $\beta_{0}$ should be predicted and selected to excite this mode. Generally, we first calculate the effective index $n_{\text {eff }}$ of the waveguide mode through the effective index method (EIM) and get a propagation constant $\beta_{1}$ at the wavelength $\lambda_{0}$. We run the FDTD solver at this $\beta$ and calculate the mode wavelength $\lambda$ by the PAT technique. Next we change the wavelength and get the new propagation constant by the EIM method, with which we run the simulation again and calculate the new mode wavelength. After several simulations a $\beta$ - $\lambda$ dispersion curve can be obtained, from which we can extract the proper propagation constant $\beta_{0}$ relative to the wavelength $\lambda_{0}$.

After the proper propagation constant is extracted, we run the FDTD solver for a relatively long time at this specific propagation constant and through the PAT technique we can calculate the mode information at the particular wavelength. In the PAT calculation the number (length) of time steps of the field component input to the PAT transform are increased from short to long and the result is recorded only when a converged value has been obtained. This is the fundamental requirement for the PAT transform to calculate any mode information. Since the mode loss is proportional to the $3-\mathrm{dB}$ bandwidth of the mode spectrum, the mode with a lower loss generally needs a higher resolution, which means a longer time sequence is needed to obtain an accurate result because the spectral resolution is generally inversely related to the length of the time sequence.

\section{A. 3-D ARROW Leaky Waveguide}

The 3-D ARROW waveguide we considered first has the same structure as in [1] which is shown in Fig. 2. The refractive indexes are 3.590 for GaAs, 3.555 for $5 \%$ AlGaAs, and 3.452 for $20 \% \mathrm{AlGaAs}$, and the operating wavelength is $1.064 \mu \mathrm{m}$. Taking the advantage of the waveguide symmetry, only half of the structure is simulated by employing a perfect electric wall

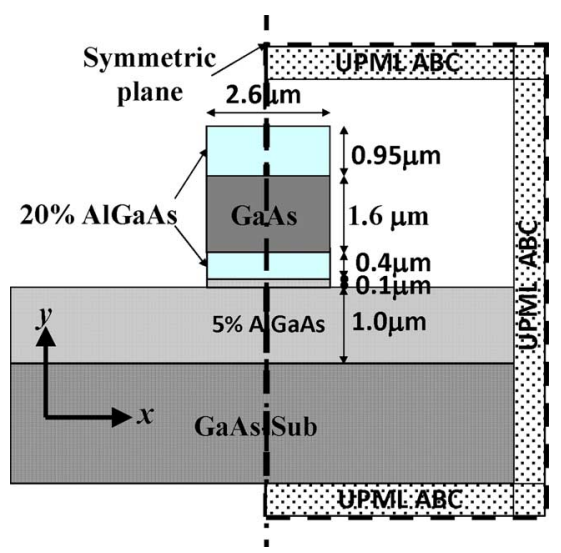

Fig. 2. Schematic structure of the cross section of the ARROW leaky waveguide.

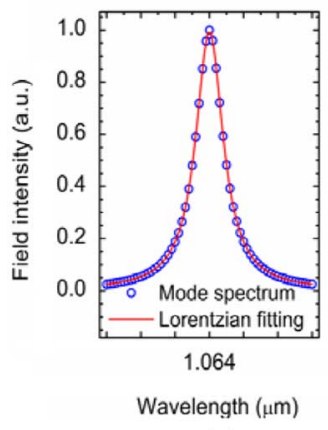

(a)

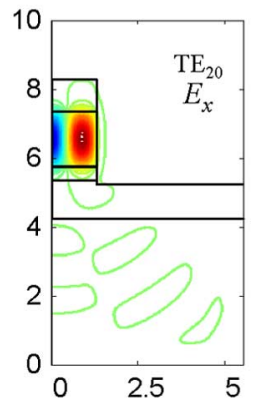

(d)

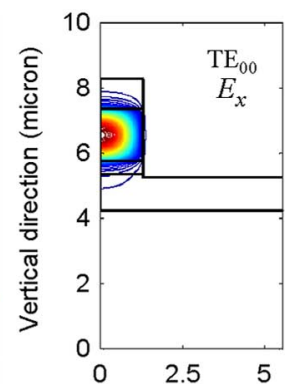

Lateral direction (micron)

(b)

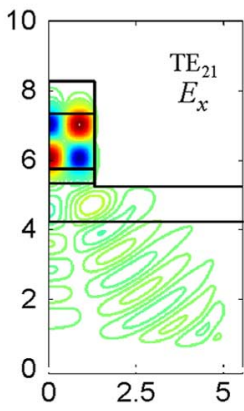

(e)

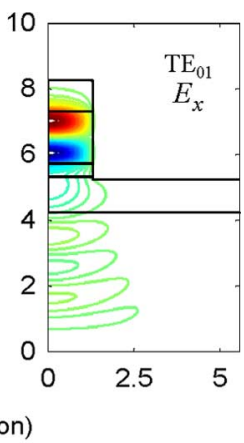

(c)

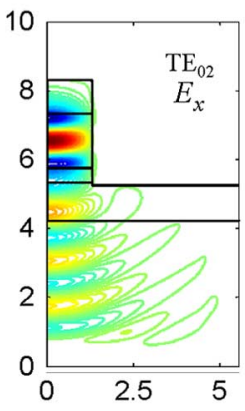

(f)
Fig. 3. (a) Calculated field intensity spectrum of the fundamental mode; (b)-(f) Contour plot of the dominant electric field distribution for $\mathrm{TE}_{01}, \mathrm{TE}_{20}, \mathrm{TE}_{21}$ and $\mathrm{TE}_{02}$ modes calculated by the built-in DFT.

at the vertical symmetry plane to simulate the first 5 symmetric transverse electric (TE) modes $\left(\mathrm{TE}_{00}, \mathrm{TE}_{01}, \mathrm{TE}_{20}, \mathrm{TE}_{21}\right.$ and $\mathrm{TE}_{02}$ ). The UPMLs with 100-cell thickness are used to terminate the computation window with a uniform space cell size of $0.0125 \mu \mathrm{m}$.

The field intensity spectrum of the fundamental mode calculated by the PAT technique from FDTD output sequences longer than 30000 after the pulse finished is stable as shown in Fig. 3(a), which shows a very good Lorentzian line shape. The contour plots of the main electric field component $E_{x}$ for these five modes are also given by the built-in DFT as shown in Fig. 3(b)-(f) for the fundamental mode as well as the first four higher order symmetric TE modes. The calculated quality factors $Q$ for these first 5 symmetric TE modes at a wavelength of $1.064 \mu \mathrm{m}$ are $1.063 \times 10^{7}, 3.321 \times 10^{4}, 3.900 \times 10^{5}, 1.693 \times 10^{4}$, 
TABLE I

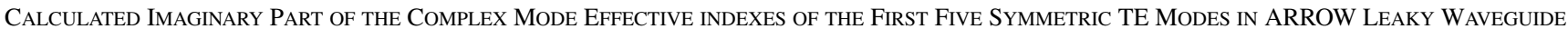

\begin{tabular}{|c|c|c|c|c|c|c|}
\hline Mode & This model & $\begin{array}{c}\text { SIM } \\
{[1]}\end{array}$ & $\begin{array}{l}\text { FEM } \\
{[2]}\end{array}$ & $\begin{array}{l}\text { Edge-element } \\
{[3]}\end{array}$ & $\begin{array}{l}\text { FEIDBPM } \\
{[5]}\end{array}$ & $\begin{array}{l}\text { IDVFEBPM } \\
{[6]}\end{array}$ \\
\hline $\mathrm{TE}_{00}$ & $\mathrm{j} 1.693 \times 10^{-7}$ & $\mathrm{j} 1.697 \times 10^{-7}$ & $\mathrm{j} 1.765 \times 10^{-7}$ & $\mathrm{j} 1.712 \times 10^{-7}$ & $\mathrm{j} 1.698 \times 10^{-7}$ & $\mathrm{j} 1.692 \times 10^{-7}$ \\
\hline $\mathrm{TE}_{01}$ & $\mathrm{j} 5.447 \times 10^{-5}$ & $\mathrm{j} 5.481 \times 10^{-5}$ & $\mathrm{j} 5.535 \times 10^{-5}$ & $\mathrm{j} 5.569 \times 10^{-5}$ & $\mathrm{j} 5.482 \times 10^{-5}$ & $\mathrm{j} 5.739 \times 10^{-5}$ \\
\hline $\mathrm{TE}_{20}$ & $\mathrm{j} 4.676 \times 10^{-6}$ & - & $\mathrm{j} 1.492 \times 10^{-6}$ & $\mathrm{j} 6.786 \times 10^{-6}$ & $\mathrm{j} 1.691 \times 10^{-6}$ & - \\
\hline $\mathrm{TE}_{21}$ & $\mathrm{j} 1.083 \times 10^{-4}$ & - & $\mathrm{j} 1.227 \times 10^{-4}$ & $\mathrm{j} 1.154 \times 10^{-4}$ & $\mathrm{j} 1.232 \times 10^{-4}$ & - \\
\hline $\mathrm{TE}_{02}$ & $\mathrm{j} 8.407 \times 10^{-4}$ & $\mathrm{j} 8.841 \times 10^{-4}$ & - & $\mathrm{j} 8.831 \times 10^{-4}$ & - & $\mathrm{j} 9.179 \times 10^{-4}$ \\
\hline
\end{tabular}

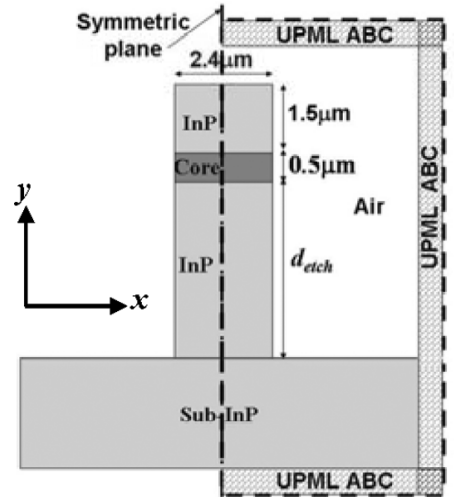

Fig. 4. Schematic structure of the simulated deep ridge waveguide.

and $2.169 \times 10^{3}$, respectively, with the corresponding group indexes $n_{g}$ calculated as $3.600,3.618,3.647,3.668$, and 3.647. Then the corresponding imaginary part of the mode effective indexes can be calculated. Results are shown in Table I and compared with other methods in the references, which shows that the difference is within $12 \%$ between our results and those obtained by the other methods for four of the five modes in Table I, and for the $\mathrm{TE}_{20}$ mode, the calculated results is in qualitative agreement [10] with other methods which typically work in the frequency domain and most of them are finite-element-based.

\section{B. 3-D Deeply Etched Ridge Waveguides}

Then we analyze a 3-D deep ridge waveguide as shown in Fig. 4 with the same structure as in [7], [10] with the refractive index of 3.2543 for the InGaAsP core with the thickness of $0.5 \mu \mathrm{m}$ at the wavelength of $1.55 \mu \mathrm{m}$. Also only half of the structure is considered by employing a perfect magnetic (electric) wall at the vertical symmetry plane to simulate the $\mathrm{TE}_{10}\left(\mathrm{TE}_{20}\right)$ mode. UPML ABCs with a $1 \mu \mathrm{m}$ thickness are applied on all the other outer boundaries with the distance of $3 \mu \mathrm{m}$ from the ridge to the PMLs for both the bottom and side boundary. A uniform space cell is used in the simulation with a size of $0.02 \mu \mathrm{m}$. In [10] it is observed that instead of continuously dropping, the loss of the $\mathrm{TE}_{10}$ mode tends to saturate when the etching depth under the waveguide core goes beyond $2 \mu \mathrm{m}$ for a ridge width of $2.4 \mu \mathrm{m}$. It is also found that the loss of both $\mathrm{TE}_{10}$ and $\mathrm{TE}_{20}$ modes tends to saturate when the etching depth is very deep for the ridge width narrower than $3 \mu \mathrm{m}$ [17]. The reason for this leaky loss saturation with a very deep etching depth is that the power carried by the minor electric field of these leaky modes in the ridge waveguide couples to the modes in the lower cladding slab waveguide, and loses energy downwards.

1) Mode Coupling Effect: When the ridge is deeply etched, the lower cladding InP layer under the waveguide core forms

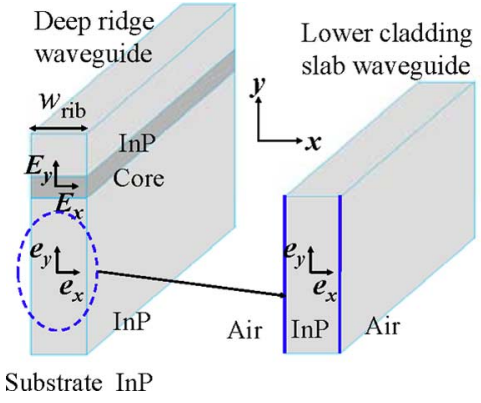

Fig. 5. Schematic diagram of the deep ridge waveguide and the lower cladding slab waveguide.

a slab waveguide confined by the air in the horizontal direction as shown in Fig. 5. The lower cladding slab waveguide has its own te and tm modes. The tm mode has the $e_{x}$ major electric field component and the $e_{z}$ minor component, and te mode only has the $e_{y}$ component. The ridge waveguide has TE and TM modes but we focus on the TE mode here. The TE mode of the ridge waveguide has the $E_{x}$ major electric component and the $E_{y}$ minor electric component. For the mode coupling to efficiently happen, the mode symmetry-matching and phase-matching conditions have to be satisfied, which means the slab modes have the same symmetry properties along the horizontal direction with the ridge waveguide modes and have an effective index not less than those of the ridge waveguide modes. For the symmetry matching, the coupling may happen between the $\mathrm{TE}_{10}$ mode of the ridge waveguide and the fundamental te mode $\left(\mathrm{te}_{0}\right)$ or first order $\mathrm{tm}$ mode $\left(\mathrm{tm}_{1}\right)$ of the lower cladding slab waveguide; or between the $\mathrm{TE}_{20}$ mode and the first-order te mode $\left(\mathrm{te}_{1}\right)$ or second-order tm mode $\left(\mathrm{tm}_{2}\right)$ of the lower cladding slab waveguide.

We use the effective index method (EIM) method to calculate the mode effective indexes of the ridge waveguide mode (for this deep ridge waveguide, the EIM method can generate modal effective indexes with high accuracy). By solving the three-layer slab waveguide in the horizontal direction, the mode effective indexes of the slab modes in the lower cladding slab waveguide can also be easily calculated. Fig. 6 shows the calculated modal effective indexes for the ridge width of 1-3 $\mu \mathrm{m}$, from which we observe that only the te $\mathrm{e}_{0}\left(\mathrm{te}_{1}\right)$ mode has an effective index larger than the $\mathrm{TE}_{10}\left(\mathrm{TE}_{20}\right)$ mode. This means the coupling will only occur between the $\mathrm{te}_{0}\left(\mathrm{te}_{1}\right)$ slab waveguide mode and the $\mathrm{TE}_{10}\left(\mathrm{TE}_{20}\right)$ ridge waveguide mode, and there will be no coupling between the $\mathrm{tm}_{1}\left(\mathrm{tm}_{2}\right)$ mode and the $\mathrm{TE}_{10}\left(\mathrm{TE}_{20}\right)$ mode. Therefore, the $E_{y}$ component of the $\mathrm{TE}_{10}\left(\mathrm{TE}_{20}\right)$ mode will couple to the te $e_{0}\left(t_{1}\right)$ mode in the lower cladding slab waveguide which will carry energy downwards no matter how deep the ridge waveguide has been etched (there will be no mode 


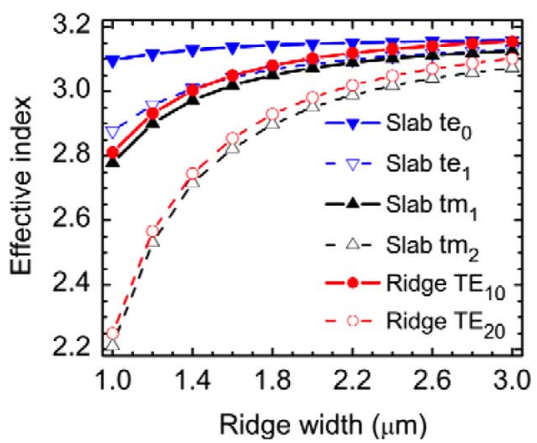

Fig. 6. Mode effective indexes of leaky modes in very deeply etched ridge waveguide and slab modes in the lower cladding slab waveguide.

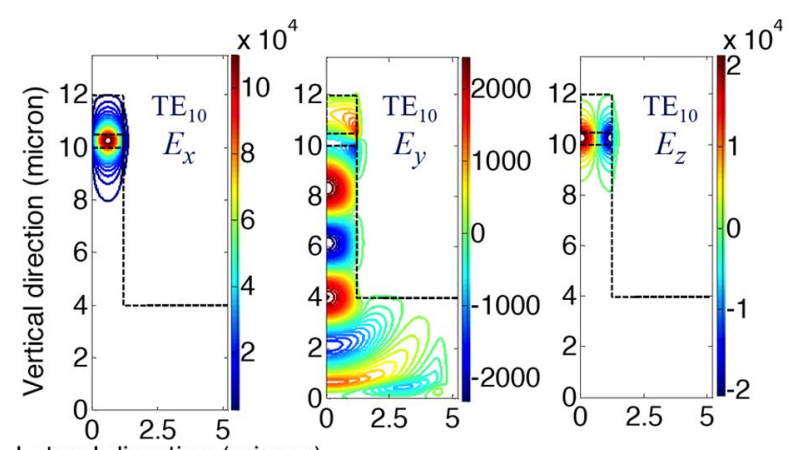

Lateral direction (micron)

(a)

(b)

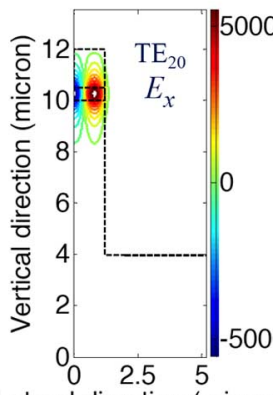

Lateral direction (micron)

(d)

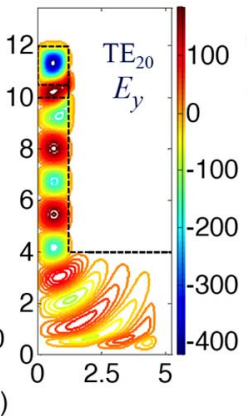

(c)

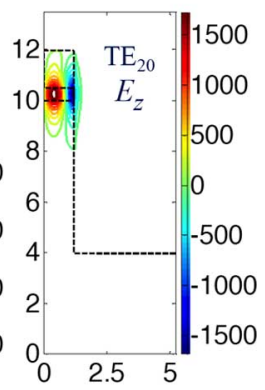

(e)

(f)

Fig. 7. Calculated field distribution of $E_{x}, E_{y}, E_{z}$ component of leaky modes in very deep ridge waveguide with a ridge width of $2.4 \mu \mathrm{m}$ for the $\mathrm{TE}_{10}$ mode from (a) to (c) and the $\mathrm{TE}_{20}$ mode from (d) to (f).

coupling for the $E_{x}$ and $E_{z}$ components). This coupling results in the leaky loss saturation behavior for the leaky modes when the etching depth under the waveguide core is very deep.

Fig. 7 shows the field component distributions obtained from the built-in DFT from 80000 FDTD iterations for the $\mathrm{TE}_{10}$ and $\mathrm{TE}_{20}$ modes in the deep ridge waveguide with an etching depth under the core of $6 \mu \mathrm{m}$ and a ridge width of $2.4 \mu \mathrm{m}$. It can be observed that the $e_{x}$ and $e_{z}$ components in the lower cladding slab waveguide are not excited, while the $e_{y}$ component is excited strongly. This confirms the above analysis.

When the coupling effects occur, the propagation constant of slab waveguide mode can provide a component matching to the propagation constant of the ridge waveguide mode and simultaneously provide another component pointing downward which carries energy away from the ridge waveguide. From the field distribution of the $E_{y}$ component shown in Fig. 7(b) and (e), the
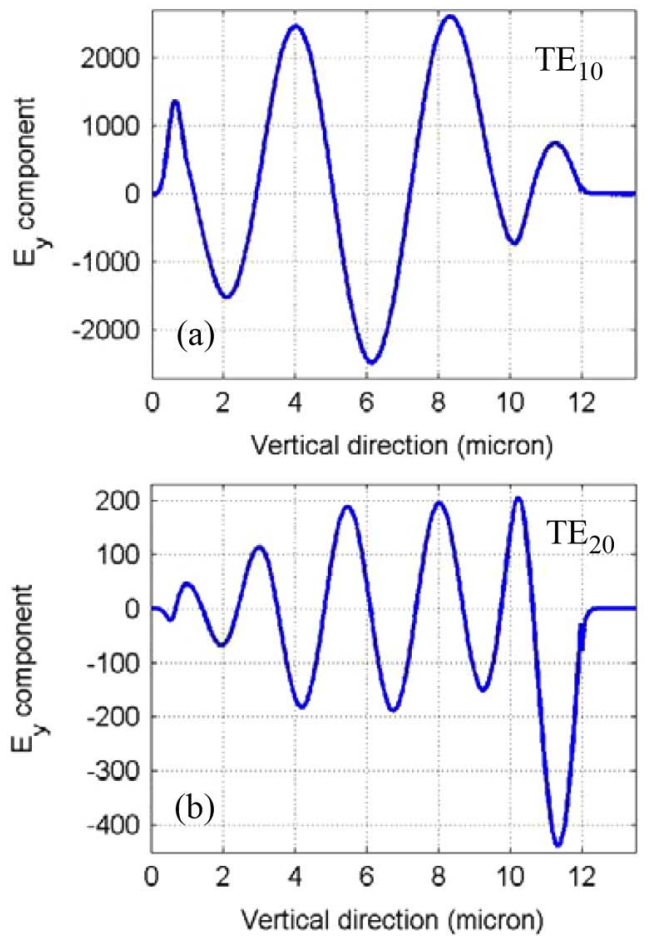

Fig. 8. Field distribution of $E_{y}$ along the vertical direction (horizontally at peak) for (a) $\mathrm{TE}_{10}$ and (b) $\mathrm{TE}_{20}$ mode in the deep ridge waveguide with an etching depth of $6 \mu \mathrm{m}$ and a ridge width of $2.4 \mu \mathrm{m}$.

mode demonstrates propagation behavior in the lower cladding slab waveguide. For $2.4 \mu \mathrm{m}$ ridge width, the mode effective indexes of the $\mathrm{TE}_{10}$ and $\mathrm{TE}_{20}$ mode at $1550 \mathrm{~nm}$ are calculated to be 3.1393 and 3.0488, respectively, and are 3.1536 and 3.1098 for the $t_{0}$ and te $e_{1}$ mode in the lower cladding slab waveguide, respectively. Therefore, according to the above explanation, the mode field should propagate in the lower cladding slab waveguide downwards with a propagation constant as

$$
\beta_{\perp}=k_{0} \sqrt{n_{\mathrm{te}_{i}}^{2}-n_{\mathrm{TE}_{i+1,0}}^{2}}, \quad i=0,1
$$

which is 1.50 and $2.49 \mathrm{rad} / \mu \mathrm{m}$ for the $\mathrm{TE}_{10}$ and $\mathrm{TE}_{20}$ mode, respectively. We plot the distribution of the $E_{y}$ component along the vertical direction (horizontally it is at peak) in Fig. 8(a) and (b) for the $\mathrm{TE}_{10}$ and $\mathrm{TE}_{20}$ mode, respectively. The peak-peak single period length $l$ in the vertical direction is around 4.26 and $2.55 \mu \mathrm{m}$ for the $\mathrm{TE}_{10}$ and $\mathrm{TE}_{20}$ mode, respectively, from the figure, which results in propagation constants around 1.47 and $2.46 \mathrm{rad} / \mu \mathrm{m}\left(\beta_{\perp}=2 \pi / l\right)$ which agree well with the above values. This confirms further that the modes excited in the lower cladding slab waveguide through mode coupling are the te $\mathrm{e}_{0}$ and te $_{1}$ mode, respectively.

2) Leakage Cancellation: By simulation it is observed that the $\mathrm{TE}_{20}$ mode could have lower loss than the $\mathrm{TE}_{10}$ mode at some specific ridge widths [17]. Next, we will investigate the influence of the ridge width on the leaky loss of these high order leaky modes. We cover a wide range of ridge widths of practical interest from 1 to $3 \mu \mathrm{m}$ with the etching depth under the waveguide core of 1,2 , and $6 \mu \mathrm{m}$. The calculated leaky loss as a function of ridge width with the etching depth from shallow to deep is given in Fig. 9(a) and (b) for the $\mathrm{TE}_{10}$ and the $\mathrm{TE}_{20}$ mode, respectively. It can be seen that generally the leaky loss 


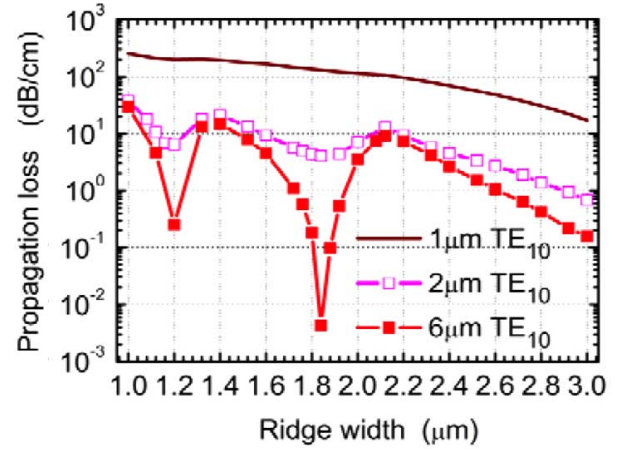

(a)

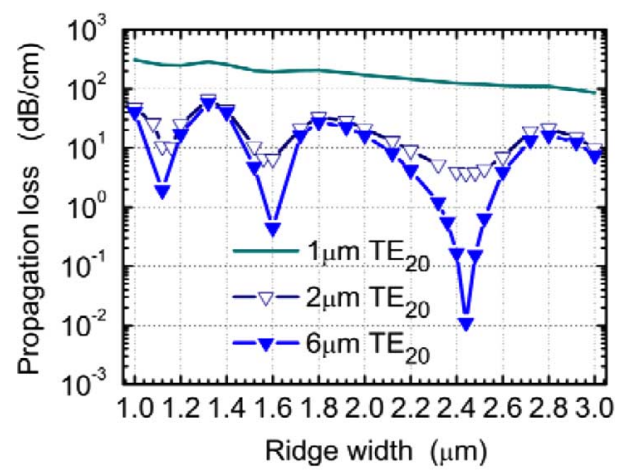

(b)

Fig. 9. Calculated propagation loss of leaky modes in a deep-ridge waveguide versus ridge width with the etching depth under the core as 1,2 and $6 \mu \mathrm{m}$ for (a) $\mathrm{TE}_{10}$ and (b) $\mathrm{TE}_{20}$ mode.

of both the $\mathrm{TE}_{10}$ and $\mathrm{TE}_{20}$ mode decreases with the ridge width increase for the three etching depths under the waveguide core. This is because the narrow ridge waveguide has a smaller mode effective index as shown in Fig. 6. From the above analysis when the ridge is really deeply etched, a lower cladding slab waveguide is subsequently formed and supports slab modes. The higher order leaky ridge mode will couple to the slab mode and loses energy downwards once the slab mode satisfies both phase-matching and horizontal symmetry-matching to the ridge waveguide mode. As shown in Fig. 6, the narrow ridge waveguide has a bigger difference of the effective refractive indexes between the coupled $\mathrm{TE}_{10}\left(\mathrm{TE}_{20}\right)$ mode and te $\left(\mathrm{te}_{1}\right)$ mode, the leaky energy will be larger than that of the wide ridge waveguide. However, from Fig. 9(a) and (b) it is also observed that at specific ridge width, the leaky loss nearly disappears. For the waveguide with etching depth under the core near $1 \mu \mathrm{m}$, the cancellation phenomena are very weak, while they become significant with the etching depth increases at the same ridge width. When the etching depth reaches $6 \mu \mathrm{m}$, the cancellation peaks become very sharp and in this case the leaky loss is very low, generally several order of magnitude lower than normal values, and the leakage at the narrow ridge width is larger than that at the wide ones. In the simulated ridge width range, the cancellation ridge widths are around 1.2 and $1.84 \mu \mathrm{m}$ for the $\mathrm{TE}_{10}$ mode, and 1.12, 1.6 and $2.44 \mu \mathrm{m}$ for the $\mathrm{TE}_{20}$ mode. This cancellation effect is due to the fact that the coupled slab mode in the lower cladding slab waveguide interferes destructively at specific ridge widths, which prevents energy leaking to the substrate.

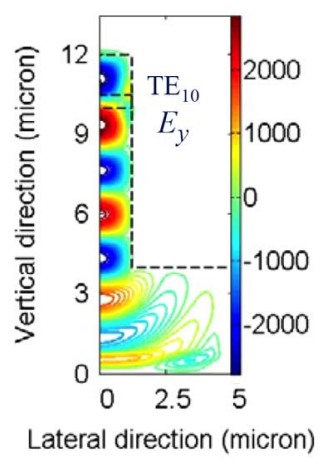

(a)

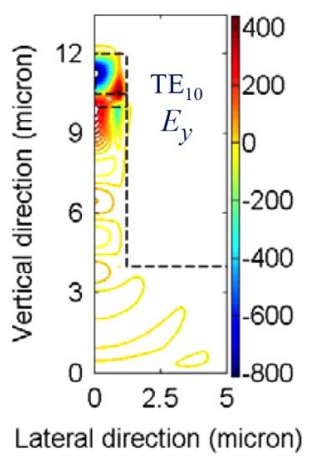

(b)
Fig. 10. Contour plot of the field distribution of the $E_{y}$ component of $\mathrm{TE}_{10}$ mode at ridge width of: (a) $2.12 \mu \mathrm{m}$ and (b) $1.84 \mu \mathrm{m}$.

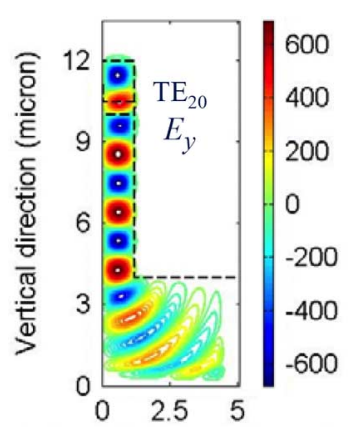

Lateral direction (micron)

(a)

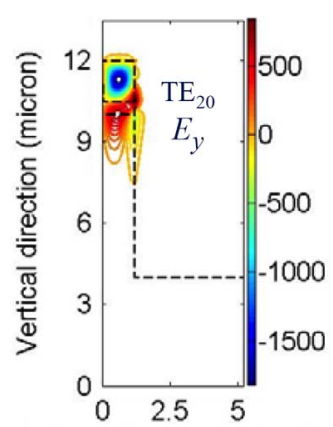

Lateral direction (micron)

(b)
Fig. 11. Contour plot of the field distribution of the $E_{y}$ component of $\mathrm{TE}_{20}$ mode at ridge width of: (a) $2.12 \mu \mathrm{m}$ and (b) $2.44 \mu \mathrm{m}$.

To understand the above leakage cancellation behavior, we analyze the mode field distributions of these two modes for very deep etching depths up to $6 \mu \mathrm{m}$. Fig. 10 shows the $E_{y}$ component distribution of the leaky modes at the cancellation and normal ridge width calculated from the embedded DFT. It can be obviously seen from Fig. 10(a) that the coupled slab mode shows strong propagation in the lower cladding slab waveguide and the energy strongly leaks to the substrate for the $\mathrm{TE}_{10}$ mode at ridge width of $2.12 \mu \mathrm{m}$, while from Fig. 10(b) the field in the slab and substrate is much weaker which means no significant coupling occurs at the cancellation ridge width of $1.84 \mu \mathrm{m}$. For the $\mathrm{TE}_{20}$ mode given in Fig. 11, at the ridge width of $2.12 \mu \mathrm{m}$ the field in the slab is strongly leaky through the slab while at the cancellation ridge width of $2.44 \mu \mathrm{m}$ the field in the slab is much weaker in (a) and (b), respectively.

Similar phenomena have been observed in [18]-[21]. In their case, the mode coupling happens in the lateral direction, however, in our case the coupling happens in the vertical direction. So it is not easy to directly take the same theory to explain our observations. The mechanism of the cancellation dependence on the ridge width in our case for the high order leaky modes is complicated. However, the fundamental mechanism could be the same: the "destructive interference" in the lower cladding causes the loss cancellation. This unwanted leakage cancellation behavior for the high-order leaky modes can deteriorate the performance of devices based on deep ridge waveguides and therefore should be carefully avoided in practical applications. 


\section{CONCLUSION}

The compact 2-D FDTD method with UPML boundary condition combined with the PAT technique has been introduced for leaky modes analysis. Compared with the frequency domain mode solvers based on the complex root searching, this model is easy to implement and can calculate the mode loss and the propagation constant separately and reliably. As a time-domain method it takes into account all mode coupling effects naturally in the simulation, which makes it very suitable to analyze leaky modes with a very low level of leakage. To validate the efficiency of this model, a perfect metal waveguide and a 3-D ARROW waveguide are analyzed. The approach is also used to analyze a 3-D deep ridge waveguide and leakage cancellation behavior for high order leaky modes in very deeply etched ridge waveguides at specific ridge widths has been observed.

\section{REFERENCES}

[1] G. M. Berry, S. V. Burke, J. M. Heaton, and D. R. Wight, "Analysis of multilayer semiconductor rib waveguides with high refractive index substrates," Electron. Lett., vol. 29, pp. 1941-1942, 1993.

[2] H. E. Hernández-Figueroa, F. A. Fernández, Y. Lu, and J. B. Davies, "Vectorial finite element modeling of 2-D leaky waveguides," IEEE Trans. Magnet., vol. 31, pp. 1710-1713, 1995.

[3] S. Selleri, L. Vincetti, A. Cucinotta, and M. Zoboli, "Complex FEM modal solver of optical waveguides with PML boundary conditions," Opt. Quantum Electron., vol. 33, pp. 359-371, 2001.

[4] W. P. Huang, C. L. Xu, W. Lui, and K. Yokoyama, "The perfectly matched layer boundary condition for modal analysis of optical waveguides: leaky mode calculations," IEEE Photon. Technol. Lett., vol. 8, pp. 652-654, 1996.

[5] Y. Tsuji and M. Koshiba, "Guided-mode and leaky-mode analysis by imaginary distance beam propagation method based on finite element scheme," J. Lightw. Technol., vol. 18, pp. 618-623, 2000.

[6] S. S. A. Obayya, B. M. A. Rahman, K. T. V. Grattan, and H. A. El-Mikati, "Full vectorial finite-element-based imaginary distance beam propagation solution of complex modes in optical waveguides," J. Lightw. Technol., vol. 20, pp. 1054-1060, 2002.

[7] M. Kohtoku, T. Hirono, S. Oku, Y. Kadota, Y. Shibata, and Y. Yoshikuni, "Control of higher order leaky modes in deep-ridge waveguides and application to low-crosstalk arrayed waveguide gratings," $J$. Lightw. Technol., vol. 22, pp. 499-508, 2004.

[8] S. Xiao, R. Vahldieck, and H. Jin, "Full-wave analysis of guided wave structures using a novel 2-D FDTD," IEEE Microw. Guided Wave Lett., vol. 2, pp. 165-167, 1992.

[9] S. Xiao and R. Vahldieck, "An efficient 2-D FDTD algorithm using real variables," IEEE Microw. Guided Wave Lett., vol. 3, pp. 127-129, 1993.

[10] Q. Lu, W. Guo, D. Bryne, and J. Donegan, "Analysis of leaky modes in deep-ridge waveguides using the compact 2-D FDTD method," Electron. Lett., vol. 45, pp. 700-701, 2009.

[11] J.-P. Berenger, "A perfectly matched layer for the absorption of electromagnetic waves," J. Computational Phys., vol. 114, pp. 185-200, 1994.

[12] S. D. Gedney, "An anisotropic perfectly matched layer absorbing medium for the truncation of FDTD lattices," IEEE Trans. Antennas Propag., vol. 44, no. 12, pp. 1630-1639, Dec. 1996.

[13] W. Guo, W. Li, and Y. Huang, "Computation of resonant frequencies and quality factors of cavities by FDTD technique and padé approximation," IEEE Microw. Wireless Compon. Lett., vol. 11, pp. 223-225, 2001.

[14] A. C. Cangellaris, "Numerical stability and numerical dispersion of a compact 2-D/FDTD method used for the dispersion analysis of waveguides," IEEE Microwave Guided Wave Lett., vol. 3, no. 1, pp. 3-5, Jan. 1993.
[15] W. Kuang, W. J. Kim, A. Mock, and J. O'Brien, "Propagation loss of line-defect photonic crystal slab waveguides," IEEE J. Sel. Top. Quantum Electron., vol. 12, no. 6, pp. 1183-1195, Nov./Dec. 2006.

[16] M. Tong and Y. Chen, "Analysis of propagation characteristics and field images for printed transmission lines on anisotropic substrates using a 2-D FDTD method," IEEE Trans. Microw. Theory Tech., vol. 46, pp. 1507-1510, 1998.

[17] Q. Lu, W. Guo, D. Bryne, and J. Donegan, "Analysis of leaky modes in very deeply etched semiconductor ridge waveguides," IEEE Photon. Technol. Lett., accepted for publication.

[18] M. A. Webster, R. M. Pafchek, A. Mitchell, and T. L. Koch, "Width dependence of inherent TM-mode lateral leakage loss in silicon-oninsulator ridge waveguides," IEEE Photon. Technol. Lett., vol. 19, no. 6, pp. 429-431, Mar. 2007

[19] T. Nguyen, R. Sekhar, M. Webster, T. Koch, and A. Mitchell, "Analysis of lateral leakage loss in silicon-on-sinsulator thin-rib waveguides," in Proc. Opto-Electron. Commun. Conf./Australian Conf. Opt. Fiber Technol., 2008, pp. 1-2.

[20] K. Ogusu, "Optical strip waveguide: A detailed analysis including leaky modes," J. Opt. Soc. Amer., vol. 73, no. 3, pp. 353-357, 1983.

[21] S. T. Peng and A. A. Oliner, "Leakage and resonance effects on strip waveguides for integrated optics," Trans. Inst. Electron. Jpn., vol. E61, pp. 151-154, 1978.

Qiaoyin Lu was born in Jiangsu Province, China, in 1974. She received the B.Sc. and M.Sc. degrees in optics from the Changchun Institute of Optics and Fine Mechanics, Changchun, China, in 1998 and 2001, respectively, and the Ph.D. degree from the Institute of Semiconductors, Chinese Academy of Sciences, Beijing, China, in 2004, studying the FDTD simulation and fabrication of photonic microcavities.

She is currently a Postdoctoral Researcher with the School of Physics, Trinity College, Dublin, Ireland. Her current research is focused on designing singlemode lasers and high-speed photodetectors.

Weihua Guo was born in Hubei province, China, in 1976. He received the B.Sc. degree in physics from Nanjing University, Nanjing, China, in 1998, and the Ph.D. degree from the Institute of Semiconductors, Chinese Academy of Sciences, Beijing, China, in 2004. His Ph.D. research was on FDTD simulation and fabrication of optical microcavities and design of semiconductor optical amplifiers.

Since September 2004, he has been a Postdoctoral Researcher with the Department of Physics, Trinity College, Dublin, Ireland. His current research is focused on optimizing microcavity two-photon absorption photodetectors and designing tunable integrated sources for coherent WDM systems.

Diarmuid C. Byrne was born in Donegal, Ireland, in 1983. He received the B.A. (mod) and Ph.D. degrees from the School of Physics, Trinity College, Dublin, Ireland, in 2005 and 2009, respectively.

His current research interests include design, characterization, and applications of widely tunable semiconductor lasers.

John F. Donegan (M'04-SM'08) received the B.Sc. and Ph.D. degrees in physics from National University of Ireland, Galway, Ireland.

He held postdoctoral positions with Lehigh University, Bethlehem, PA, and the Max Planck Institute, Stuttgart, Germany. He is a Professor of physics with Trinity College, Dublin, Ireland. His research interests are in photonic structures including broadly tunable lasers based on etched slots, microcavity two-photon absorption detectors, spherical microcavity structures, and photonic molecules. $\mathrm{He}$ also studies the interaction of quantum dots with human macrophage cells.

Prof. Donegan is a Fellow of the Institute of Physics and the Institute of Nanotechnology and a member of the American Physical Society. 